\title{
Preface to the special issue on Content-Based Multimedia Indexing
}

\author{
Stéphane Marchand-Maillet • Patrick Lambert • \\ Bernard Mérialdo • Jenny Benois-Pineau
}

Published online: 16 February 2013

(C) Springer Science+Business Media New York 2013

Content-Based Multimedia Indexing (CBMI) is the multi-disciplinary field broadly aiming at extracting knowledge from multimedia information and providing fluent access to it. CBMI research proposes to develop methods to model, abstract and organise multimedia data in view of fast, effective and accurate access. CBMI covers all the chain from the data to the user. It encompasses a large variety of challenges from multimedia information capture and representation via content understanding and information fusion to effective information indexing and retrieval. Since the early days, CBMI research has therefore produced a wide range of fundamental and application-oriented contributions and has provided methodological guidelines for progressing in the field. Due to its multi-disciplinary nature, CBMI research has also strongly been impacted by progress in adjunct domains such as machine learning, large-scale information storage and processing, and new modes of user interaction.

The International Workshop on Content-based Multimedia Indexing has been established as one of the most important forums for the presentation of the newest ideas and original research results in the field. Following the eight successful previous events of CBMI (Toulouse 1999, Brescia 2001, Rennes 2003, Riga 2005, Bordeaux 2007, London 2008, Chania 2009, Grenoble 2010 and Madrid 2011), CBMI 2012, the 10th edition of the workshop was organised by the LISTIC lab of the University of Savoie, in Annecy, France from June 27th to 29th, 2012. CBMI 2012 brought together

S. Marchand-Maillet $(\varangle)$

Department of Computer Science, University of Geneva, Geneva, Switzerland

e-mail: stephane.marchand-maillet@unige.ch

P. Lambert

LISTIC, Polytech Annecy-Chambéry, Annecy, France

B. Mérialdo

EURECOM, Sophia Antipolis, France

J. Benois-Pineau

University of Bordeaux1, LABRI UMR 5800, Bordeaux, France 
the various communities involved studying in the different aspects of the contentbased multimedia indexing, retrieval, browsing and presentation issues. The scientific program of CBMI 2012 included invited keynote talks and regular and special sessions with contributed research papers. CBMI 2012 received 54 submissions from over 17 countries (49 papers and five demo proposals) from which the program committee selected 24 for oral presentations, 14 for poster presentations and five for demo presentations.

This special issue of the MTAP journal was created to publish both new extended versions of CBMI 2012 submitted papers and also completely new contributions. In response to the open call, 19 papers were submitted and after rigorous reviewing according to the journal rules, six of them were finally accepted for publication.

The special issue is structured to successively visit all aspects of CBMI. The first two papers study multimedia content representation. "Video Fingerprinting Based on Graph Model" by Xiushan Nie et al. propose a model where two graph models are constructed for keyframe selection and foreground extraction, respectively. From a graph-based representation of the video, keyframes are selected and used to identify the foreground in frames of the corresponding shots. "Retina Enhanced SURF Descriptors for Spatio-Temporal Concept Detection" by Sabin Tiberius Strat et al. propose to apply a human retina model to preprocess video sequences before constructing the State-Of-The-Art Bag-of-Word analysis.

An advanced formal study of multimedia content understanding is proposed in "Boosted Kernel for Image Categorization" by Alexis Lechervy et al. Authors propose a framework to learn an effective kernel function using the Boosting paradigm to linearly combine weak kernels and then use a SVM with such kernels to categorize image databases.

Indexing is addressed by the next two papers. "Efficient Binary Code Indexing with Pivot based Locality Sensitive Clustering" by Wei Zhang et al. presents the Pivot Based Locality Sensitive Clustering (PLSC) along with Density Adaptive Binary coding (DAB) in PLSC clusters. This contribution aims at designing an efficient indexing based on binary code clustering and where only codes in estimated relevant clusters are scanned. "Distributed Media indexing based on MPI and MapReduce" by Hisham Mohamed et al. proposes an adapted structure of the MapReduce programming model using MPI for multimedia indexing. The aim is to provide a fundamental distributed indexing framework suited to adapt to the large volumes of data inherent to CBMI applications.

Finally "Retrieval of High-Dimensional Visual Data: current state, trends and challenges ahead" by Antonio Foncubierta-Rodríguez et al. systematically reviews current techniques in various fields of 3D and 4D visual information retrieval and analyses the currently dominating application areas. This paper provides a clear insight on future challenges in the field.

We warmly thank all those who have helped to accomplish the task of successfully organizing this special issue of MTAP and CBMI 2012. We want to thank specially the technical chairs of the conference (Shin'ichi Satoh and Stéphane MarchandMaillet) and the reviewers of both the conference and this special issue. Their contribution was the basis for selecting the high quality of the works presented here. We also thank all the authors of the papers submitted to the workshop and to this special issue; they constitute the true force behind the success of the workshop and are the first accountable for the quality and the interest of this issue. 


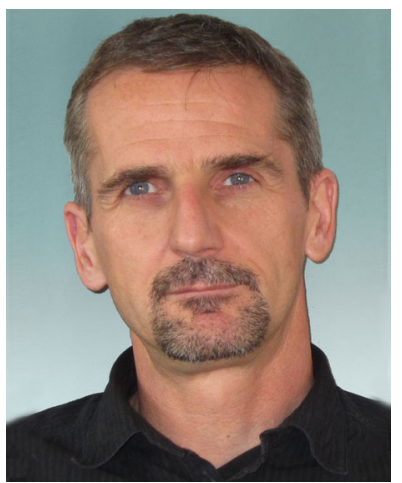

Stéphane Marchand-Maillet is Associate Professor in the Department of Computer Science at University of Geneva. He holds a PhD in Applied Mathematics from Imperial College UK ('97). He is the founding leader of the Viper Research group on Information Retrieval and Machine Learning. $\mathrm{He}$ has authored a number of journal and conference contributions on Information Retrieval, Machine Learning, Data Mining and Multimedia processing. He was general chair of the ACM SIGIR 2010 in Geneva, CH and ACM CIVR 2009 in Santorini, GR. He is involved in a number national and international projects, in relation to Information Access. He participates in several scientific committees, including as Chair of the Technical Committee 12 of the International Association for Pattern Recognition ('Multimedia and Visual Information Systems'). http://viper.unige.ch/stephane

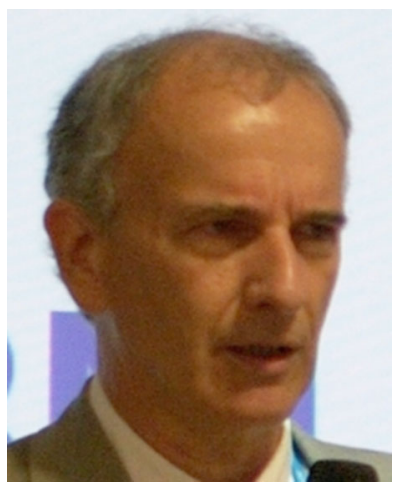

Patrick Lambert received the enginer degree in electrical engineering in 1978, and the PhD degree in signal processing in 1983, both from the National Polytechnic Institute of Grenoble, France. He is currently a Full Professor at the School of Engineering of University of Savoie, Annecy, France and a member of the Informatics, Systems, Information and Knowledge Processing Laboratory (LISTIC), Annecy, France. His research interests are in the field of image and video analysis, and actually dedicated to non linear color filtering and video semantic indexing. 


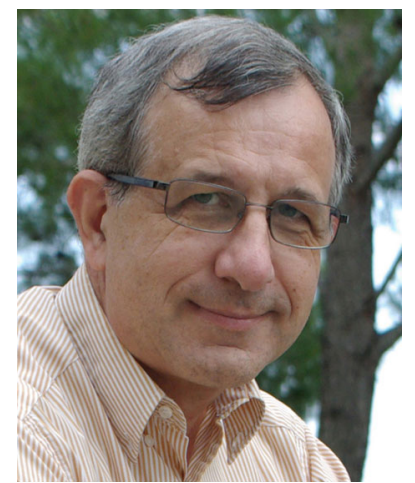

Bernard Mérialdo is professor in the Multimedia Department of EURECOM, France and current head of the department. A former student of the Ecole Normale Supérieure, Paris, he received a $\mathrm{PhD}$ from Paris 6 University and an "Habilitation à Diriger des Recherches" from Paris 7 University. For more than 10 years, he was a research staff, then project manager at the IBM France Scientific Center, working on probabilistic techniques for Large Vocabulary Speech Recognition. He later joined EURECOM to set up the Multimedia Department. His research interests are the analysis, processing, indexing and filtering of Multimedia information to solve user-related tasks. His research covers a whole range of problems, from content extraction based on recognition techniques, content understanding based on parsing, multimedia content description languages (MPEG7), similarity computation for applications such as information retrieval, user personalization and user interaction for the design of innovative applications. He participates in numerous conference program committees. He is part of the organizing committee for the CBMI workshop series. He was editor for the IEEE Transactions on Multimedia and general chair of the ACM Multimedia conference in 2002. He often acts as an expert and reviewer for French and European research programs. He is a Senior Member of IEEE and member of ACM.

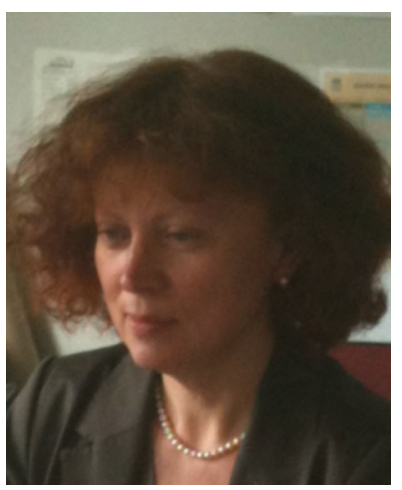

Jenny Benois-Pineau is a distinguished professor (classe exceptionnelle) of Computer science at the University Bordeaux 1 and chair of Video Analysis and Indexing research group in Image and Sound Department of LABRI UMR 58000 UniversitéBordeaux1/Bordeaux2/CNRS/ENSEIRB. She is also a deputy scientific director of theme B of French national research unity GDR CNRS ISIS, and director of Computer Science Department of the faculty of Mathematics and Computer science at the University Bordeaux 1. She obtained her PhD degree in Signals and Systems in Moscou and her Habilitation à Diriger la Recherche in Computer Science and Image Processing from University of Nantes France. Her topics of interest include image and video analysis and indexing, motion analysis and content description for content-based multimedia retrieval. She is the author and co-author of more than 130 papers in international journals, conference proceedings, book chapters. She has 
tutored an co-tutored $20 \mathrm{PhD}$ students and 19 research masters. She is associated editor of EURASIP Signal Processing:Image Communication, Elsevier, Multimedia Tools and applications, Springer, TS Hermes-Lavoisier journals. She has served in numerous program committees in international conferences and workshops: ACM MM, CIVR, CBMI, AMR, IPTA, SAMT, ECMCS... She has served as expert for European Commission since FP4 and is a member of Technical Advisory group for Media programme EACEA DG Culture, CE. She is a member of Multimedia Commission of French Ministry of National Education and member of scientific board of International Center for Mathematical Modelling at the University of Växjo, Sweden. She has been coordinator or leading researcher in international research projects Platon, Balaton, IP XMedia, French representative in COST292 European action, national research projects ANR and numerous projects with French industrial companies. She gave invited lectures at the universities of Sussex (GB), UPC (Spain), UNAM (Mexico), University of North Carolina at Chapel Hill, Brooklynn Polytechnic, NJIT, CMU(USA), Florence (Italy), Kuala Lumpur (Malasia), Veszprem (Hungary). 